\title{
ENDEMIC MACROLEPIDOPTERA SUBSPECIES IN THE NATURAL HISTORY MUSEUM COLLECTIONS FROM SIBIU (ROMANIA)
}

\author{
SERGIU-CORNEL TÖRÖK, GABRIELA CUZEPAN
}

\begin{abstract}
The paper presents data regarding endemic Macrolepidoptera subspecies preserved in the Entomological Collections of Natural History Museum from Sibiu. 22 endemic subspecies are recorded and represented by 382 specimens in the Entomological Collection. Most of the specimens have been collected from mountain habitats, especially from Southern and Western Carpathians. The results of this paper contribute to the improvement of the existing data concerning the distribution and outline the areas of Macrolepidoptera's endemism in Romania.
\end{abstract}

Résumé. Le document présente des données concernant les sous-espèces endémiques des Macrolépidoptères conservées dans les collections entomologiques du Musée d'Histoire Naturelle de Sibiu. 22 sous-espèces endémiques sont enregistrées et représentées par 382 spécimens dans la collection entomologique. La plupart des spécimens ont été recueillis dans les habitats de montagne, en particulier du Sud et l'Ouest des Carpates. Les résultats de cette étude contribuent à compléter les données existantes concernant la distribution et de définir les zones d'endémisme des Macrolépidoptères en Roumanie.

Key words: Macrolepidoptera, endemic taxa, geographic distribution, museum collections.

\section{INTRODUCTION}

In this paper, the authors wish to present the endemic taxa from the Natural History Museum from Sibiu. The term endemic is used for taxa that are unique to a geographic location. This geographic location can be either relatively large or very small (Gaston \& Spicer, 1998; Kenyeres et al., 2009).

In Romania, we encounter 88 endemic taxa (Rákosy, 1997); of which an important proportion is occupied by the Macrolepidoptera group, represented by 59 endemic species and subspecies (Burnaz, 2008).

In the Catalogue of the Romanian Lepidoptera (Rákosy et al., 2003) the taxonomic status of some endemic Macrolepidotera was changed. Namely, Limenitis populi bucovinensis (Hormuzachi, 1897), Neptis rivularis latefasciata Hassler \& Weber, 1982, Melanargia galathea niculescui Varin, 1965 are considered infraspecific variations of these taxa. Other subspecies had been synonymised. Erebia aethiops fogarasica Warren, 1931 and Erebia aethiops mesorubria PopescuGorj, 1955 are actually Erebia aethiops aethiops (Esper, 1777), E. pharte belaensis Goltz, 1937 is a synonym of E. pharte carpatina Popescu-Gorj \& Szabó, 1986 and E. pharte romaniae Hormuzachi, 1937 was described after some aberrant forms of $E$. epiphron transsylvanica Rebel, 1908. Regarding this last remark Cuvelier \& Dincă (2007) considered that E. pharte romaniae Hormuzachi, 1937 was erroneously mistaken with Erebia sudetica (Staudinger, 1861).

In recent publications, Euphydryas maturna opulenta Rákosy \& Varga, 2012 was described from North Dobrogea. This subspecies has distinctive external and genital characteristics. The subspecific separation was proved by the genetic studies (Rákosy et al., 2012). 
The zoogeographical distribution of the Romanian endemic taxa shows that the majority of endemic taxa are found in the Carpathian Mountains. The presence of numerous Erebia sp. (E. epiphron transsylvanica, E. pharte belaensis, E. manto traianus, E. gorge fridericikoenigi, and E. pronoe regalis) and Glacies sp. (G. coracina dioszeghyi, G. noricana carpathica and G. canaliculata schwingenschussi) is most remarkable (Rákosy, 1997; Varga, 2003).

Erebia melas subspecies inhabit only island-like calcareous mountains rocks, so E. melas carpathicola is found only in the Hășmaș and Ciucaș Mountains and E. melas runcensis can be encountered only in Apuseni Mountains (Rákosy, 1997; Varga, 2003). In addition to these taxa, other endemic taxa are widespread in the Carpathians and in the surrounding mountainous areas as Aricia artaxerexes issekutzi Balogh, 1956 and Photedes captiuncula delattini Varga, 1970 (Varga, 2003).

Besides the Carpathians, in Romania we encounter other endemic rich areas. In Băile Herculane - Cazanele Dunării area 11 endemic taxa are present, and in Dobrogea, 10 endemic taxa (Rákosy, 1997).

On these grounds, the Carpathian Mountains, especially the eastern and southern parts, together with the mountains of western Transylvania (Apuseni and Banat Mountains), Băile Herculane - Cazanele Dunării and Dobrogea are considered core areas of survival and autochthonous evolution in many invertebrate groups (Varga, 2003).

From these territories, but also from other areas, in the Entomological Collections of the Natural History Museum from Sibiu, endemic Lepidoptera taxa are preserved. The paper is presenting the endemic subspecies preserved in the Entomological Collection of the Natural History Museum from Sibiu. Through this study new data regarding the Macrolepidoptera endemic subspecies is being added in regard to Romania's fauna. The presented data outlines the zones of butterflies' endemism in Romania.

Until now, numerous researches have been made regarding the Entomological Collections of the Natural History Museum from Sibiu. At the beginning of the $20^{\text {th }}$ century Daniel Czekelius wrote at least 12 papers regarding the Lepidoptera from Transylvania, all of these papers basing on the Entomological Collections of the Natural History Museum from Sibiu. Also, Victor Weindel, Eugen Worell and Eckbert Schneider wrote about the Lepidoptera from Transylvania. Eckbert Schneider also compiled the Catalogues of Victor Weindel and Heinrich Hann von Hannenheim Entomological Collections (Schneider, 1984, 2003). The endemic taxa specimens from these two collections were analysed for this article once again.

Several identification keys were used: for Pieridae, Nymphalidae, Lycaenidae - Tolman \& Lewington (2009) and Niculescu (1961, 1963, 1965), for the Nymphalidae, Satyrinae species - Varga $(1999,2002)$, for the endemic Geometridae - Leraut (2009) and for the Erebidae - Leraut (2006). Unfortunately, no endemic Noctuidae species or subspecies have been found in the museum's collection.

\section{MATERIAL AND METHODS}

The analyzed material is part of the Entomological Collections of Natural History Museum from Sibiu, comprising over 265,000 of insect specimens. The endemic Macrolepidoptera subspecies belong to several collections as follows: Daniel Czekelius Lepidoptera Collection from Transylvania, Victor Weindel Lepidoptera Collection from Transylvania, Eugen Worell Entomological Collection, Heinrich 
Hann von Hannenheim Entomological Collection, Rolf Weyrauch Entomological Collection and Eckbert Schneider Entomogical Collection.

Daniel Czekelius Lepidoptera Collection, counting 7,162 specimens of Macro- and Microlepidoptera from Transylvania, is considered to be one of the most valuable collections from the Natural History Museum from Sibiu, being dated 1887. Daniel Czekelius became a member of the Transylvania Society (Siebenbürgische Verein für Naturwissenschaften zu Hermannstadt) in 1887, the year when he was chosen also curator of the Entomological Collection (Pascu \& Schneider, 1998). His collection reflects, through its preserved specimens, the Lepidoptera fauna from Transylvania.

Victor Weindel Lepidoptera's Collection from Transylvania, Eugen Worell's Entomological Collection, Heinrich Hann von Hannenheim's Entomological Collection and Rolf Weyrauch's Entomological Collection contain insect specimens collected during the period 1920-1970, from many parts of Romania, but especially from Transylvania Region (Pascu \& Schneider, 1998).

Some parts of the endemic Macrolepidoptera specimens, preserved in the museum collection, have been sampled by other collectors as follows: Emil von Silbernagel, Sachsenheim, Karl Petri, Béla Cseh, László Diószeghy, Friedrich Deubel, Köntzey Gerő, Paul Tiltscher, Ştefan Péterfi, Arnold Müller, Josef v. Mallász, Adriano Ostrogovich, Béla Kiss, Friedrich König, Aurelian Popescu-Gorj, Mircea Brătăşeanu. The most recent samples of the Lepidoptera specimens have been made by Eckbert Schneider during the period 1950-1990.

The material was collected from mountainous area, especially from the Southern and Western Carpathians.

The nomenclature and systematical list is presented according to the one used in The Catalogue of the Romanian Lepidoptera (Rákosy et al., 2003) and de Jong (2010).

The endemic taxa are listed according to their appearance in the collections. For each endemic subspecies the data of the specimen's labels is given (as follows: sampling site, date, collector's name). For the sampling sites the original name and toponymy are given, as well as the administrative territory or mountain range.

The following abbreviations were used in this paper:

ESC $=$ Eckbert Schneider Entomological Collection; DCLCT $=$ Daniel Czekelius Lepidoptera Collection from Transylvanian; EWC = Eugen Worell Entomological Collection; HHC = Heinrich Hann von Hannenheim Entomological Collection; RWC = Rolf Weyrauch Entomological Collection; VWLT $=$ Victor Weindel Lepidoptera Collection from Transylvanian .

A.P.G. = Aurelian Popescu-Gorj; A.Mü. = Arnold Müller; A.O. = Adriano Ostrogovich; B.K. = Béla Kiss; Bartha $=$ Viktor von Bartha; C.B. = Béla Cseh; Cz. = Daniel Czekelius; Dannehl = F. Dannehl; E.S./ E.A.S.= Eckbert Schneider; Wo. = Eugen Worell; F.Kö. = Frederic König; F.D. = Friedrich Daubel/Deubel [The older papers are citing this collector as Daubel (Czekelius, 1922; Diószeghy, 1933-1934), and the newer as Deubel (Pascu \& Schneider, 1998; Rákosy, 1996)]; H.P. = Hans Pelits; H.H. = Heinrich Hann von Hannenheim; J.M = Josef v. Mallász; Kö. = Köntzei Geró; L.D. = László Diószeghy; M.B. = Mircea Brătăşeanu; S.P. = Ştefan Péterfi; K.P. = Karl Petri; R.W. $=$ Rolf Weyrauch; E.Silb. $=$ Emil von. Silbernagel; P.T. $=$ Paul Tiltscher; A.Ma. = A. Marcu; B. and A. Lipthay $=$ Bela Lipthay and A. Lipthay; Da. = Dannehl; E.F. = Eduard Fleck; M.K. = Moritz von Kimakowicz.

Alt. = altitude; det. = determinated by; leg. = collector; Anonymous = anonymous collector; Mt./ Mts $=$ Mountain/s; $\mathrm{n} .=$ number of the specimen in Czekelius Collection; spec. $/$ specs $=$ specimen $/$ specimens.

Abbreviations of the county names in Romania:

AB - Alba; BV - Brașov; CS - Caraș Severin; CV - Covasna; GL - Galați; HD - Hunedoara; HR Harghita; NT - Neamț; PH - Prahova; SB - Sibiu; VL - Vâlcea. 
From the Lepidoptera Collection of Natural History Museum from Sibiu, 22 endemic Macrolepidoptera subspecies are presented below, represented by 382 specimens.

\section{RESULTS}

Family Pieridae

Subfamily Pierinae

Pieris bryoniae carpathensis Moucha, 1956

ESC: 1 spec. Moldoveanu Geb. [Făgăraş Mts], 06.1954., leg. E.S.; 1 spec. Arpăşel Valley [Făgăraş Mts], 27-30.07.1976, leg. E.S.; 1 spec. Cheile Dâmbovicioarei [Piatra Craiului Mts], 02.08.1978, leg. E.S.; 1 spec. Königstein [Piatra Craiului Mts], 25.07.1967, leg. E.S.; 2 specs Mţii. Făgăraş, Valea Sâmbăta [Făgăraş Mts, Sâmbăta Valley, BV], 27.06.1976, leg. E.S.; 5 specs Mții. Făgăraş, Valea Sâmbăta [Făgăraş Mts, Sâmbăta Valley, BV], 22.06.1965, leg. E.S.; 6 specs Mţii. Vrancei, Valea Lepşei 1050 m [Lepşa Valley, Vrancei Mts], 11.06.1974, leg. E.S.

RWC: 1 spec. Ceahlău [Ceahlău Massif, NT], 25.06.1954, leg. R.W.; 1 spec. Bâlea [Bâlea Valley, Făgăraş Mts], 07.1953, leg. R.W.

EWC: 1 spec. Bâlea [Bâlea Valley, Făgăraş Mts], 07.1943, leg. Wo.

VWLT [P. bryoniae O. wolenskyi Berger]: 1 spec. Mt. Bătrâna [Bătrâna Mt., Cindrel Mts], 05.08.1925, leg. Anonymous; 8 specs Mt. Postăvaru [Postăvaru Massif, Postăvaru Peak, BV], 06.07.1954, leg. Anonymous; 1 spec. Mt. Bucegi, Vârful cu Dor [Vârful cu Dor, Bucegi Mts, PH], 04.07.1955, leg. Anonymous.; 1 spec. Mt. Bucegi, Vârful cu Dor [Vârful cu Dor, Bucegi Mts, PH], 07.07.1955, leg. Anonymous.

HHC [P. bryoniae O. wolenskyi Berber]: 1 spec. Podragu, $2100 \mathrm{~m}$ [Podragu Mt., Făgăraş Mts], 30.05.1954, leg. Anonymous; 1 spec. Sâmbăta [Sâmbăta Valley, BV], 22.07.1957, leg. Anonymous; 1 spec. Mănăstirea Sâmbăta [Sâmbăta Monastery, BV], 07.08.1964, leg. Anonymous.

Family Lycaenidae

Subfamily Polyommatinae

Polyommatus dorylas magnus Czekelius, 1917

(Fig. $1 \mathrm{~A}, \mathrm{~B})$

DCLCT [= Lycaena hylas v. magnus]: 1 spec. Bòrnek [Borsec, HR], 22.07.1911, leg. Cz.; 1 spec. Gyorgyó Szent Miklos [Gheorgheni, HR], 12.07.1912, leg. P.T.; 1 spec. Ghylkos-tó [Lacu Roşu, HR], 18.07.??, leg. Cz.; 1 spec. Bòrnek [Borsec, HR], 15.07.1911, leg. Cz.; 1 spec. Bòrnek [Borsec, HR], 08.08.1910, leg. Cz.

\section{Pseudophilotes bavius hungaricus Diószeghy, 1913}

(Fig. 1 C, D)

RWC: 1 spec. Zakelberg [Zakel hill, Slimnic, SB], 09.05.1954, leg. R.W.; 1 spec. Zakelberg [Zakel hill, Slimnic, SB], 18.05.1954, leg. R.W.; 1 spec. Zakelberg [Zakel hill, Slimnic, SB], 19.04.1961, leg. R.W.

ESC: 1 spec. Zakelberg [Zakel hill, Slimnic, SB], 05.05.1956, leg. Wo.; 1 spec. Zakelberg [Zakel hill, Slimnic, SB], 14.05.1955, leg. E.S. 

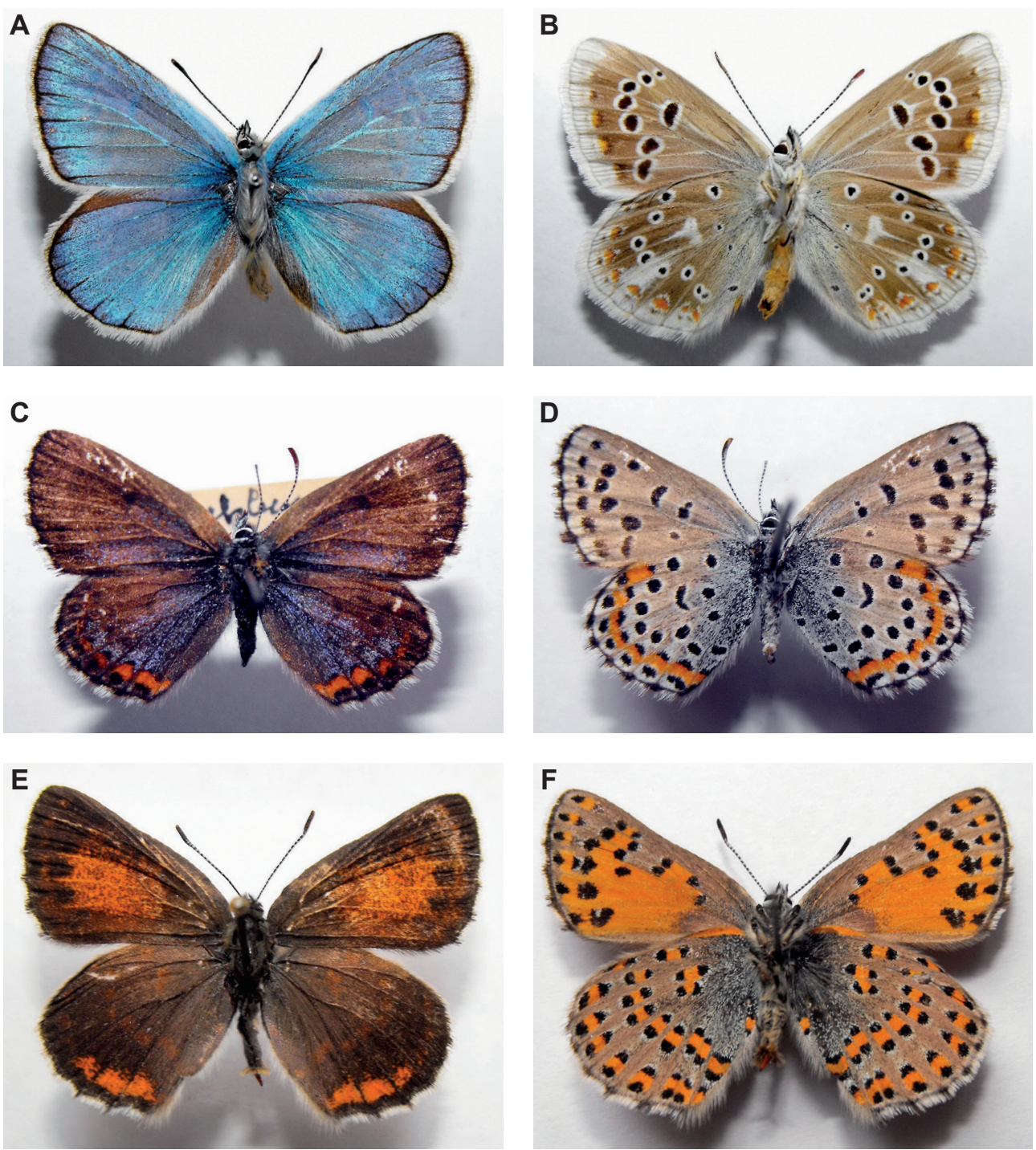

Fig. 1 - Polyommatus dorylas magnus: A, dorsal view; B, ventral view; Pseudophilotes bavius hungaricus: $\mathrm{C}$, dorsal view; D, ventral view; Tomares nogelii dobrogensis: $\mathrm{E}$, dorsal view; $\mathrm{F}$, ventral view (Photos: Sergiu C. Törok \& Gabriela Cuzepan).

\section{Subfamily Theclinae}

Tomares nogelii dobrogensis Caradja, 1895

(Fig. 1 E, F)

RWC: 1 spec. Pădurea Gârboavele, Galați [Gărboavele forest, GL], 03.06.1975, leg. A.Ma.; 1 spec. Pădurea Gârboavele, Galaţi [Gărboavele forest, GL], 15.06.1978, leg. R.W.

ESC: 2 specs Girbeav [Gărboavele forest, GL], 16.06.1975, leg. Anonymous; 1 spec., without data. 


\section{Family Nymphalidae \\ Subfamily Satyrinae \\ Erebia epiphron transsylvanica Rebel, 1908}

(Fig. 2 A, B)

RWC: 4 specs Retezat [Retezat Mts], 08.1961, leg. R.W.; 10 specs Königstein [Piatra Craiului Mts], 07.1963, leg. R.W.; 3 specs Lotru Geb. [Lotru Mts], 1920.07.1960, leg. R.W.; 6 specs Vânturariţa [Buila-Vânturariţa National Park, VL], 16-18.07.1960, leg. R.W.

EWC: 2 specs Parâng, 2000 m [Parâng Mts], 21.08.1932, leg. Wo.; 1 spec. without data; Piatra Arsă, Bucegi, 1600 m [Piatra Arsă, Sinaia, PH], 23.08.1946, leg. A.P.G.; 1 spec. Bucegi, Babele, 2100 m [Babele, Bucegi Mts, PH], 23.08.1946, leg. A.P.G.; 1 spec. Bucegi, Casa Schill [Bucegi Mts, Schill House, PH], 20.08.1938, leg. Anonymous; 1 spec. Retezat, 1800-2000 m [Retezat Mts, 1800-2000 alt.], 28.07.1926, leg. L.D.

HHC: 1 spec. Bâlea 1400 m [Bâlea, Făgăraş Mts], 12.08.1958, leg. Anonymous; 1 spec. Tarita 2100 m [Tăriţa, 2100 alt., Făgăraş Mts], 14.08.1958, leg. Anonymous; 1 spec. Tarita 2100 m [Tărița, 2100 alt., Făgăraş Mts], 15.08.1958, leg. Anonymous; 1 spec. Chica Pietrelor 1400 m [Chica Pietrelor, Făgăraş Mts], 26.07.1963, leg. Anonymous.

ESC: 2 specs Mţii. Retezat, Vârful Peleaga [Retezat Mts, Peleaga Peak], 06.08.1958, leg. E.S.; 3 specs Mţii. Retezat, Valea Pietrelor [Retezat Mts, Pietrelor Valley], 04.08.1958, leg. E.S.; 2 specs Mţii. Retezat, Creasta Slăvei-Judele [Retezat Mts, Slăvei-Judele Ridge], 05.08.1958, leg. E.S.; 5 specs Mţii. Retezat, Rezervaţia Naturală, 2000 m [Retezat Mts, 2000 m alt., Nature Reserve], 08.08.1974, leg. E.S.; 1 spec. Mţii. Retezat, Circul Galeşul [Retezat Mts, Galeşul Cirque], 03.08.1958, leg. E.S.; 1 spec. Mţii. Retezat, Valea Galeşului [Retezat Mts, Galeşului Valley], 03.08.1958, leg. E.S.; 5 specs without data; 1 spec. Piatra Craiului [Piatra Craiului Mts], 03.08.1978, leg. E.A.S.; 10 specs Mții. Făgăraş, Valea Ionel-Fedeleş [Fedeleş Valley, Făgăraş Mts], 25.07.1976, leg. E.S.; 4 specs Mţii. Făgăraş, Valea Arpăşel [Făgăraş Mts, Arpăşel Valley], 27-30.07.1976, leg. E.S.

\section{Erebia manto trajanus Hormuzachi, 1895}

RWC: 1 spec. Retezat [Retezat Mts], 22.07.1971, leg. F.Kö.

EWC [E. manto v. mantoides ??]: 2 specs Herkulesbad [Băile Herculane, CS], 24.07.1936, leg. Wo.

ESC: 1 spec. Vârful Postăvaru [Postăvaru Massif, Postăvaru Peak, BV], 02.08.1963, leg. E.S.; 6 specs Mt. Căpăţâni, Briota [Căpăţânii Mts, Briota Peak], 1519.07.1960, leg. E.S.

Erebia sudetica radnaensis (Rebel, 1915)

RWC [Erebia sudetica Staudinger, 1861 ssp. ??]: 1 spec. Ciucaş, Mt. Roşu [Muntele Roşu, Ciucaş Mts, PH], 27.07.1962, leg. A.P.G.; 2 specs Mt. Retezat, Vf. Zănoaga [Retezat Mts, Zănoaga Peak], 03.08.1969, leg. H.P., 1 spec. Mt. Retezat, Stâna Zlatna, 1700 m [Retezat Mts, Zlatna Sheepfold], 24.07.1967, leg. F.Kö.

EWC [Erebia melampus sudetica ??]: 1 spec. Retezat [Retezat Mts], 12.07.1928, leg. L.D.; $1 \mathrm{spec}$. without data.

ESC: 3 specs Mţii. Retezat, Gura Apei [Gura Apei, Retezat Mts], 19.07.1967, leg. E.S. 
A

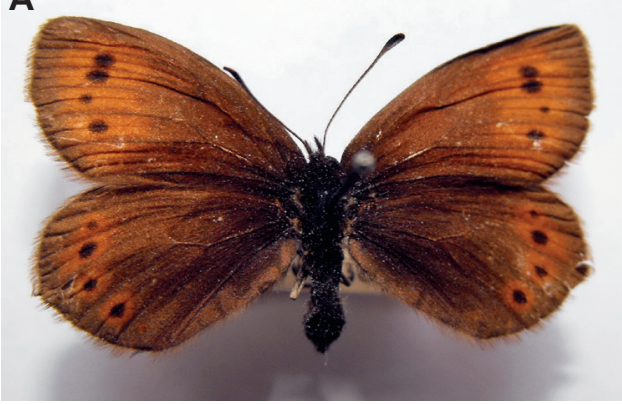

C
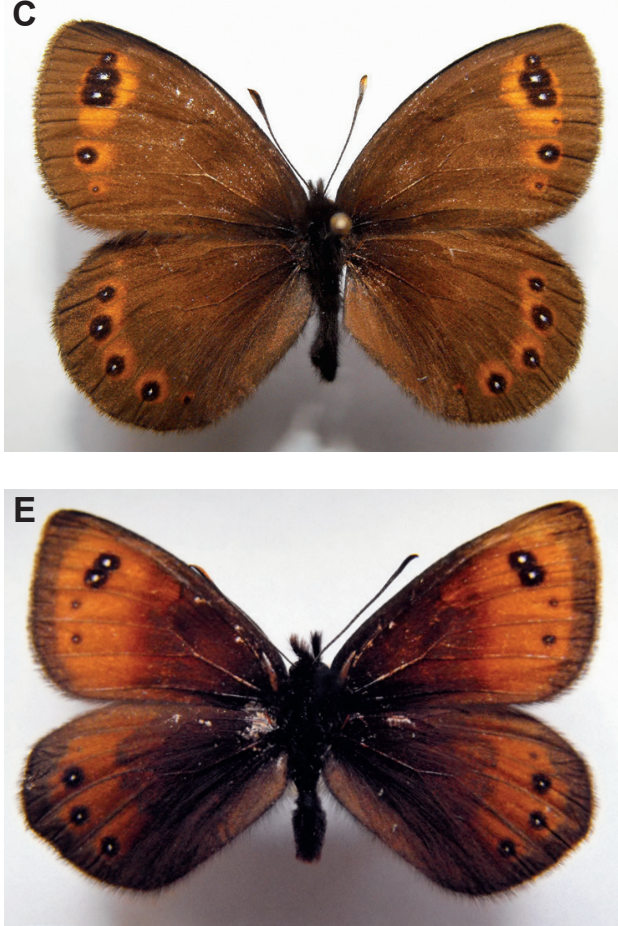

B

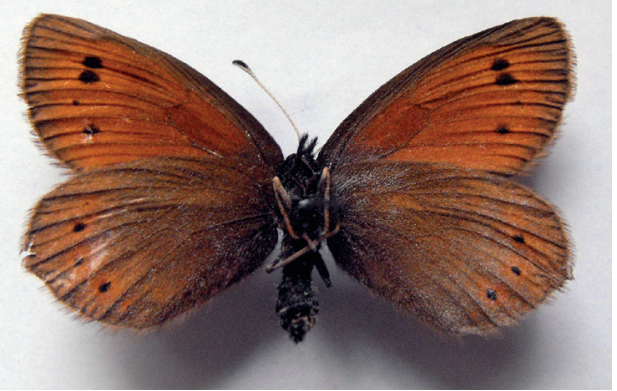

D

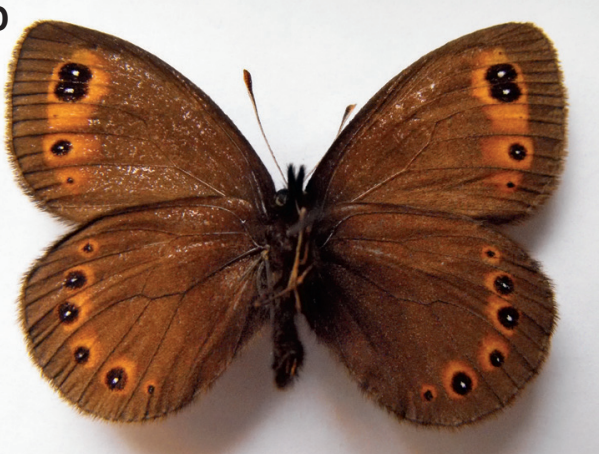

$\mathbf{F}$

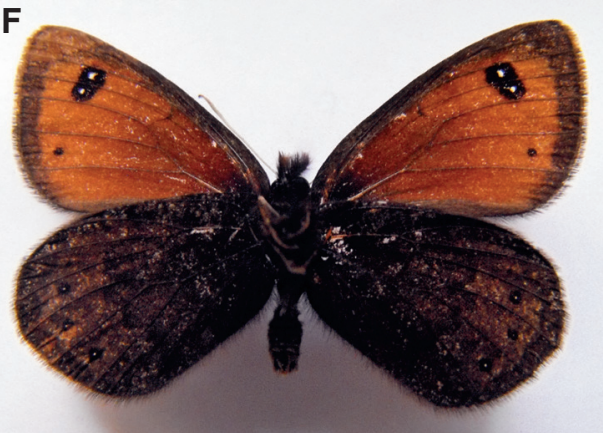

Fig. 2 - Erebia epiphron transsylvanica: A, dorsal view; B, ventral view; Erebia medusa psodea: C, dorsal view; D, ventral view; Erebia gorge fredericikoenigi: E, dorsal view; F, ventral view (Photos: Sergiu C. Törok \& Gabriela Cuzepan).

Erebia medusa psodea (Hübner, 1804)

(Fig. 2 C, D)

RWC: 3 specs Herkulesbad [Băile Herculane, CS], 07.06.1970, leg. R.W.; 1 spec. Herkulesbad [Băile Herculane, CS], 10.06.1970, leg. R.W.; 2 specs Herkulesbad [Băile Herculane, CS], 06.06.1970, leg. R.W.; 1 spec. Herkulesbad [Băile Herculane, CS], 13.06.1970, leg. R.W.; 2 specs Herkulesbad [Băile Herculane, CS], 1018.06.1975, leg. R.W.; 1 spec. Herkulesbad [Băile Herculane, CS], 28.06.1956, leg. R.W.; 1 spec. Herkulesbad [Băile Herculane, CS], 30.06.1956, leg. R.W.; 2 specs Domogled [Domogled-Cerna Valley National Park], 09.06.1970, leg. R.W.; 1 spec. 
Domogled [Domogled-Cerna Valley National Park], 12.06.1970, leg. R.W.; 1 spec. Domogled [Domogled-Cerna Valley National Park], 18.06.1964, leg. R.W.; 1 spec. Domogled [Domogled-Cerna Valley National Park], 20.06.1964, leg. R.W.

VWLT: 1 spec. Păltiniş, Stâna Muncel [Păltiniş, Muncel Sheepfold, Cindrel Mts], 08.07.??, leg. Cz.; 2 specs Mt. Bucegi, Vârful cu Dor [Vârful cu Dor, Bucegi Mts, PH], 04.07.1955, leg. Anonymous; 3 specs Postăvaru [Postăvaru Mts], 06.07.1954, leg. Anonymous.

ESC: 1 spec. Retezat, Cab. Baleia, $1450 \mathrm{~m}$ [Baleia Shelter, Retezat Mts], 23.06.1957, leg. E.S.; 1 spec. Retezat [Retezat Mts], 24.06.1957, leg. E.S.; Mt. Cozia, $1600 \mathrm{~m}$ [Cozia Mts], 17-20.06.1969, leg. E.S.; 1 spec. without data.

\section{Erebia cassioides neleus (Freyer, 1833)}

RWC: 2 specs Retezat [Retezat Mts], 07.1958, leg. R.W.; 1 spec. Mt. Arjana [Arjana Mt., Cernei Mts], 18.08.1964, leg. F.Kö.; 1 spec. Mt. Retezat, Vf. Zănoaga [Retezat Mts, Zănoaga Peak], 03.08.1969, leg. H.P.

EWC: 1 spec. without data, leg. F.Kö.; 1 spec. without data.

\section{Erebia gorge fredericikoenigi Varga, 1999}

\section{(Fig. 2 E, F)}

RWC: 1 spec. Retezat [Retezat Mts], 07.1961, leg. R.W.; 9 specs Königstein [Piatra Craiului Mts], 07.1963, leg. R.W.; 1 spec. Băile Geoagiu, Mt. Șeptar [Băile Geoagiu, Metaliferi Mts, HD], 07.1961, leg. R.W.

EWC: 4 specs without data; 1 spec. Retyezat [Retezat Mts], 18.08.1933, leg. B. and A. Lipthay; 1 spec. Parâng, 2000 m [Parâng Mts], 22.08.1932, leg. Wo.

VWLT [E. gorge pirinica Buresch]: 4 specs Mt. Surul [Surul Mt., Făgăraş Mts], 18.07.1951, leg. Anonymous.

ESC [E. gorge pirinica ??]: 2 specs Mţii. Retezat, Creasta Slăvei-Judele [Retezat Mts, Slăvei-Judele Ridge], 05.08.1958, leg. E.S.; 3 specs Mţii. Făgăraş, Valea Ionel-Fedeleş [Fedeleş Valley, Făgăraş Mts], 25.07.1976, leg. E.S.; 1 spec. Mţiii. Retezat, Peleaga [Retezat Mts, Peleaga Peak], 06.08.1958, leg. E.S.; 1 spec. Mţii. Retezat [Retezat Mts], 06.08.1958, leg. E.S.

\section{Erebia pandrose roberti Peschke, 1920}

RWC: 20 specs Pujorel, Rodna Geb. [Rodna Mts], 26.06.1955, leg. R.W.

EWC: 3 specs Pujorel, Rodna Geb. [Rodna Mts], 26.06.1955, leg. R.W.

HHC: 1 spec. Rodna-Gebirge, Mt. Galațiului, 1900 m [Galațiului Mt., 1900 alt., Rodna Mts], 15.08.1958, leg. Anonymous, det. A.P.G.

\section{Erebia pandrose cibinica Dannehl, 1927}

RWC: 1 spec. Hohe Rinne [Păltiniş, SB], 26.06.1970, leg. R.W.; 2 specs Parâng [Parâng Mts], 03.07.1956, leg. R.W.; 1 spec. Bărcaciu [Bărcaciu, Făgăraş Mts], 18.07.1956, leg. R.W.

HHC: 1 spec. Podragu, 2100 m [Podragu Mt., Făgăraş Mts], 10.07.1954, leg. Anonymous.

EWC: 3 specs Mţii. Făgăraş, Bâlea [Bâlea Valley, Făgăraș Mts], 14.07.1943, leg. Wo.; 3 specs without data; 1 spec. Retyezat [Retezat Mts], 27.08.1933, leg. B. and A. Lipthay.

VWLT: 2 specs Mt. Cindrel [Cindrel Mt., Cindrel Mts], 08.07.??, leg. Cz.; 1 spec. Mt. Beşineu [Beşineu Mt., Cindrel Mts], 28.06.1908, leg. Anonymous; 3 specs 
Mt. Bătrâna [Bătrâna Mt., Cindrel Mts], 05.08.1925, leg. Anonymous; 1 spec. Mt. Surul [Surul Mt., Făgăraş Mts], 18.07.1951, leg. Anonymous; 1 spec. without data.

ESC: 1 spec. Mţii. Retezat, 1800-1900 m [Retezat Mts], 24.06.1957, leg. E.S.; 1 spec. Mţii. Retezat, Creasta Slăvei-Judele, $2400 \mathrm{~m}$ [Retezat Mts, Slăvei-Judele Ridge], 05.08.1958, leg. E.S.; 1 spec. without data; 4 specs Mţii. Făgăraş, Scărişoara [Scărişoara Peak, Făgăraş Mts], 01.07.1981, leg. E.S.; 2 specs Mt. Căpăţâni, Briota [Căpăţânii Mts, Briota Peak], 15-19.07.1960, leg. E.S.

Erebia melas carpathicola Popescu-Gorj \& Alexinschi, 1959

RWC: 1 spec. Mt. Öcsém, Carp. Orientali [Hăşmaș Mts], 11.08.1962, leg. F. Kö.

\section{Erebia melas runcensis König, 1965}

RWC: 1 spec. Cheile Runcului, Gilău Geb. [Cheile Runcului, AB], 19.07.1972, leg. H.P.; 1 spec. Runc. [Cheile Runcului, AB], 12.07.1971, leg. F.Kö.; 2 specs Cheile Vălişoarei-Aiud [Trascău Mts, AB], 16.07.1973, leg. H.P.

ESC: 5 specs Apuseni [Apuseni Mts], 29.06.1975, leg. E.S.; 1 spec. Cheile Vălişoarei-Aiud [Trascău Mts, AB], 16.07.1973, leg. E.S.; 1 spec. Cheile Runcului, Ocolișel, Mţii. Gilău [Cheile Runcului, Ocoliş Village, AB], 19.07.1977, leg. E.S.; 2 specs Cheile Runcului, Apuseni [Cheile Runcului, Ocoliş Village, AB], 29.06.1975, leg. E.S.

\section{Erebia pronoe regalis Hormuzachi, 1937}

RWC: 7 specs Königstein [Piatra Craiului Mts], 07.1963, leg. R.W.

Subfamily Heliconiinae

Boloria pales carpathomeridionalis Crosson \& Popescu-Gorj, 1963

(Fig. 3 A, B)

DCLT: 2 specs Podragu [Podragu, Făgăraş Mts], 10.08.1904, E.F.; 1 spec. Vurtop [Vârtop, Făgăraş Mts], 06.08.??, leg. Cz; 1 spec. Fedelesch [Fedeleș Mt., Făgăraş Mts], 29.06.1910, leg. Cz; 1 spec. Fedelesch [Fedeleș Mt., Făgăraş Mts], 02.07.1911, leg. Cz; 1 spec. Fedelesch [Fedeleș Mt., Făgăraş Mts], 13.06.1909, leg. Cz; 1 spec. Fedelesch [Fedeleș Mt., Făgăraș Mts], 19.06.1927, leg. Cz; 1 spec. Butcsecs [Bucegi Mts], 04.08.??, leg. M.K.; 1 spec. Butcsecs [Bucegi Mts], 09.10.?1910, leg. F.D.

RWC: 9 specs Königstein [Piatra Craiului Mts], 07.1963, leg. R.W.; 1 spec. Piatra Arsă, Sinaia, 1850 m [Piatra Arsă, Sinaia, 1850 alt. PH], 20.08.1945, leg. A.P.G.; 1 spec. Bucegi, Babele, 2220 m [Babele, 2220 alt., Bucegi Mts, PH], 24.08.1974, leg. A.P.G.; 1 spec. Bucegi, Caraiman 2000 m [Caraiman, 2000 alt., Bucegi Mts], 31.08.1969, leg. M.B.

HHC: 3 specs Chica Pietrelor 1400 m [Chica Pietrelor, Făgăraş Mts], 26.07.1963, leg. Anonymous.

VWLT: 5 specs Mt. Surul [Suru Mt., Făgăraş Mts], 18.07.1957, leg. Anonymous.

ESC: 1 spec. Bucegi, Coştila 2300 m [Coştila Peak, 2300 alt., Bucegi Mts, PH], 30.08.1970, leg. E.S.; 1 spec. Bucegi, Caraiman 1900 m [Caraiman Peak, 1900 alt., Bucegi Mts], 12.08.1972, leg. E.S.; 1 spec. Piatra Caraiului [Piatra Caraiului Mts], 3.08.1978, leg. E.S.; 1 spec. Mţii. Făgăraş, Valea Ionel-Fedeleş [Fedeleş Valley, Făgăraş Mts], 25.07.1976, leg. E.S. 

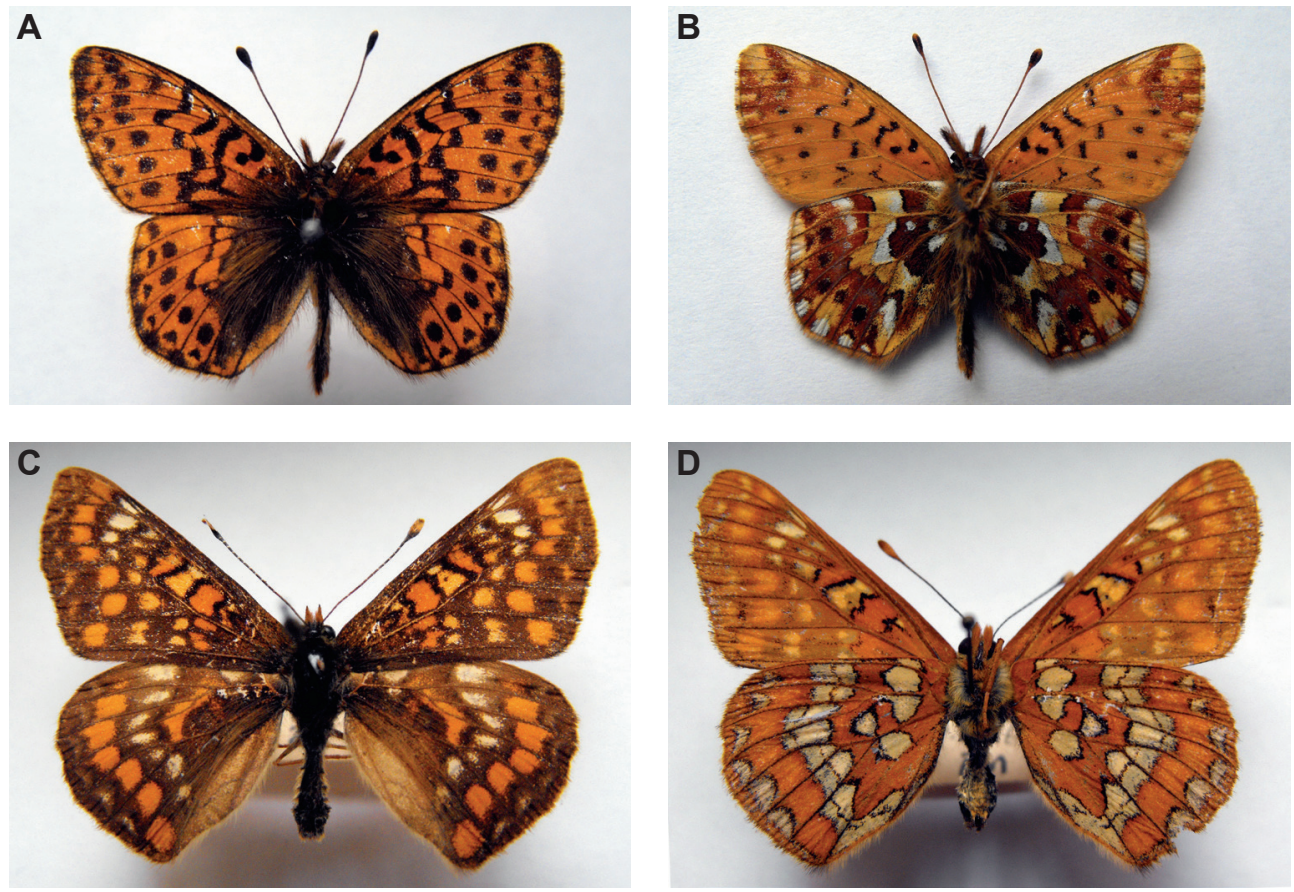

Fig. 3 - Boloria pales carpathomeridionalis: A, dorsal view; B, ventral view; Euphydryas maturna partiensis: C, dorsal view; D, ventral view (Photos: Sergiu C. Törok \& Gabriela Cuzepan).

Subfamily Melitaeinae

Euphydryas maturna partiensis (Varga, 1973)

(Fig. 3 C, D)

RWC: 2 specs Domogled [Domogled-Cerna Valley National Park], 30.06.1956, leg. R.W.; 2 specs Domogled [Domogled-Cerna Valley National Park], 26.06.1964, leg. R.W.; 1 spec. Herkulesbad [Băile Herculane, CS], 10-18.06.1973, leg. R.W.; 3 specs Domogled [Domogled-Cerna Valley National Park], 25.06.1964, leg. R.W.; 2 specs Domogled [Domogled-Cerna Valley National Park], 20.06.1956, leg. R.W.; 1 spec. Domogled [Domogled-Cerna Valley National Park], 30.06.1964, leg. R.W.; 1 spec. Domogled [Domogled-Cerna Valley National Park], 23.06.1964, leg. R.W.; 1 spec. Domogled [Domogled-Cerna Valley National Park], 29.06.1956, leg. R.W.; 1 spec. Herkulesbad [Băile Herculane, CS], 29.06.1956, leg. R.W.

ESC: 3 specs Chiş-chiş [Chichiş, CV], 22.05.1960, leg. E.S.

\section{Family Geometridae}

Subfamily Ennominae

Glacies canaliculata schwingenschussi Wehrli, 1919

(Fig. 4 A)

DCLCT [= Psodos schwingenschussi]: 1 spec. Catiave, Făgăraş Gebirge [Catiavei Summit, Făgăraş Mts], 06.08.1910, leg. Cz., det. Bartha; 1 spec. Cheia Bandes, Fogăraş Gebirge, 2300 m [Cheia Bandei, Făgăraş Mts], 07.08.1910, leg. Cz., det. Bartha; 1 spec. Parângul [Parâng Mts], without data, leg. K.P., det. Bartha; 1 spec. Retyezat [Retezat Mts], 10.07.1912, leg. F.D., det. Bartha; 5 specs Retyezat 
[Retezat Mts], 07.1928, leg. L. D., det. Bartha; 1 spec. Retyezat [Retezat Mts], 17.07.1908, leg. L.D., det. Bartha.

EWC [= Psodos schwingenschussi]: 6 specs Făgăraş Geb., Bulea [Bâlea, Făgăraş Mts], 07.1943; leg. Wo.; 2 specs Retezat, 2000 m [Retezat Mts], 22.07.1935, leg. B. and A. Lipthay.

ESC: 3 specs Mţii. Retezat, Rezervaţia Naturală, 2000 m [Retezat Mts, Nature Reserve 2000 alt.], 08.08.1974, leg. E.S.

Glacies noricana carpathica Schwingenschuss, 1915

DCLCT: 1 spec. Cheia Bandes, Fogăraş Gebirge, 2300 m [Cheia Bandei, 2300 alt. Făgăraş Mts], 07.08.1910, leg. Cz., det. Bartha.

\section{Glacies coracina dioszeghyi Schmidt, 1930}

(Fig. 4 B)

DCLCT [= Psodos dioszeghyi]: 2 specs Şerbota, 1800 m [Şerbota Summit, 1800 alt., Făgăraş Mts], 31.06.1920, leg. Anonymous, det. Bartha; 1 spec. Cheia Bandes, Fogăraş Gebirge, 2300 m [Cheia Bandei, 2300 alt., Făgăraş Mts], 07.08.1910, leg. Cz.; 1 spec. Catiave, Făgăraş Gebirge, 2200 m [Catiavei Summit, 2200 alt., Făgăraş Mts], 07.08.1910, leg. Cz.; 1 spec. Kuhhorn [Ineu Peak, Rodna Mts] 16.07.1930, leg. A.Mü., det. Bartha; 1 spec. Retyezat [Retezat Mts], 10.07.1912, leg. F.D.; 1 spec. Barkacs, Făgăraş Gebirge [Bărcaciu Hut, Făgăraş Mts], 26.07.1934, leg. Cz.; 6 specs Hohe Rinè, 1800 m [Păltiniş, SB], 15.08.1933, leg. Cz.; 1 spec. Parângul [Parâng Mts], 11.??.1934, leg. K.P.; 1 spec. Tartar, 1850 m [Tataru Summit, 1850 alt., Bucegi Mts], 09.07.1922, leg. H.H.; 2 specs Retyezat [Retezat Mts], 07.1928, leg. L.D.

EWC [= Psodos coracina; Psodos dioszeghyi]: 2 specs without data; 1 spec. Parâng, 1700-1900 m [Parâng Mts, 1700-1900 alt.], 30.07.1952, leg. Anonymous; 1 spec. Parâng, 2000 m [Parâng Mts, 2000 alt.], 12.07.1954, leg. Anonymous; 1 spec. Parâng, 1800-2100 m [Parâng Mts, 1800-2100 alt.], 10.07.1954, leg. Anonymous; 6 specs Făgăraş Geb., Bulea [Bâlea Mt., Făgăraş Mts], 07.1943, leg. Wo.; 1 spec. Mândra, Petroşani [Mândra Lake, Parâng Mts], 08.1932, leg. Wo.; 3 specs Beşineu, Hohe Rinne [Beşineu Mt., Cindrel Mts], 01.08.1941, leg. Wo.

VWLT: 1 spec. Mt. Beşineu [Beşineu Mt., Cindrel Mts], 24.05.??, leg. Anonymous; 2 specs Mt. Surul [Surul Mt., Făgăraş Mts], 18.07.1921, leg. Anonymous.

RWC: 3 specs Făgăraş Geb., Scara [Scara Mt., Făgăraş Mts], 28.6.1963, leg.

R.W.; 1 spec. Königstein [Piatra Craiului Mts], 07.1963, leg. R.W.; 1 spec. Parâng [Parâng Mts], 03.07.1956, leg. R.W.

ESC: 2 specs Mţii. Făgăraş, Valea Ionel-Fedeleş [Fedeleş Valley, Făgăraş Mts], 25.07.1976, leg. E.S.

\section{Elophos dilucidaria carphatica (Soffner, 1932)}

(Fig. $4 \mathrm{C}$ )

DCLCT [= Gnophos dilucidaria carphatica]: 1 spec. Tresnita, Fogăraş Gebirge [Trăsnita Peak, Făgăraş Mts], 07.08.1910, leg. Cz., det. A.P.G.; 1 spec. Hohe Rinè [Păltiniş, SB], 01.08.??, leg. Cz., det. A.P.G.

EWC: 1 spec. Hohe Rinne [Păltiniș, SB], 17.07.1935, leg. Anonymous; 1 spec. Hohe Rinne [Păltiniş, SB], 07.??, leg. Cz.; 1 spec. Mt. Rarău [Rarău Mts], 31.07.1953, leg. Anonymous;

VWLT: 1 spec. Şanta [Şanta, Cindrel Mts], 17.07.??, leg. Cz.; 1 spec. Lacu Roşu (Ghilcoş) [Red Lake, HR], 09.07.1954, leg. Anonymous. 

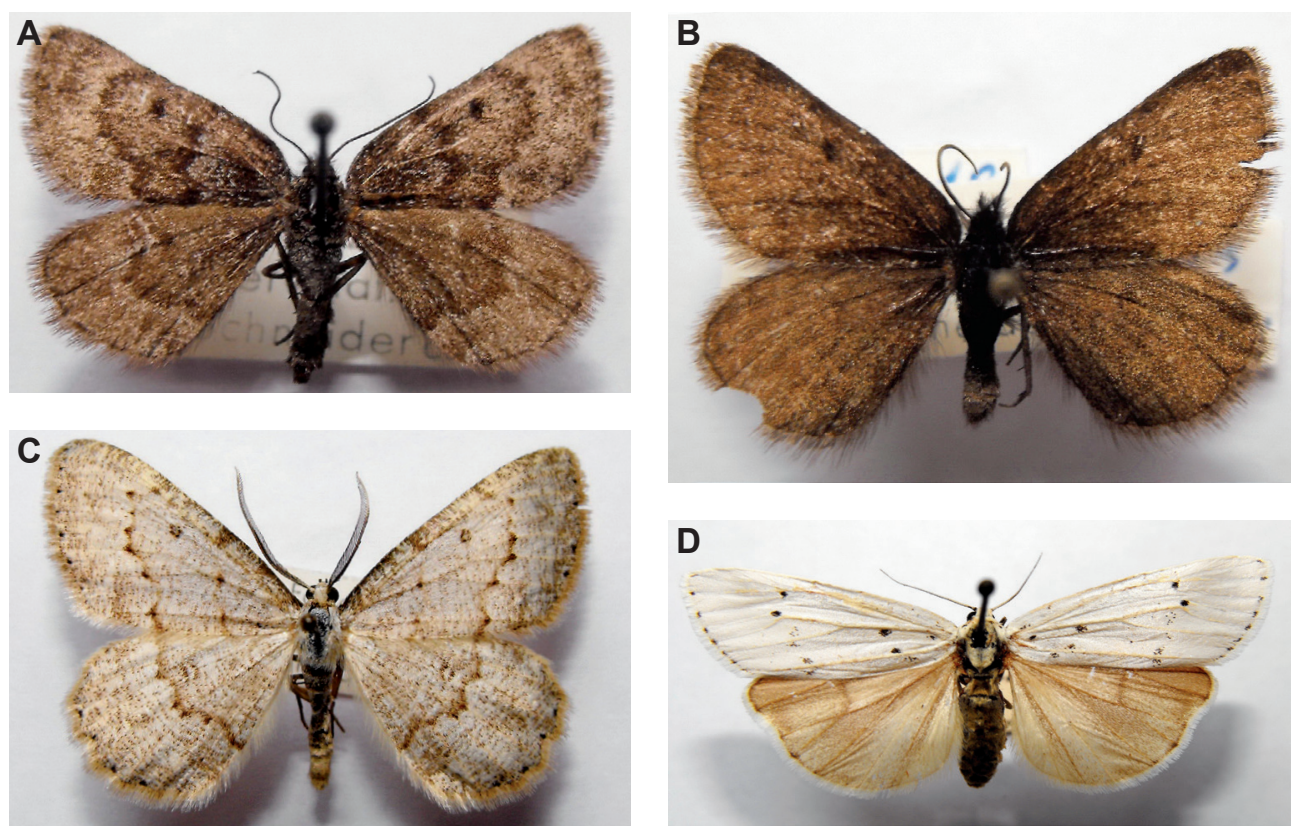

Fig. 4 - A, Glacies canaliculata schwingenschussi; B, Glacies coracina dioszeghyi; C, Elophos dilucidaria carphatica; D, Coscinia cribraria pannonica (Photos: Sergiu C. Törok \& Gabriela Cuzepan).

ESC: 3 specs Mții. Făgăraş, Valea Ionel-Fedeleş [Fedeleş Valley, Făgăraş Mts], 25.07.1976, leg. E.S.; 2 specs Piatra Craiului [Piatra Craiului Mts], 03.08.1978, leg. E.S.

Family Erebidae

Subfamily Arctiinae

Coscinia cribraria pannonica Daniel, 1955

(Fig. 4 D)

DCLCT [= Coscinia cribrum]: 1 spec. Riu Sadu [Riu Sadu, Cindrel Mts, SB], 25.07.1913, leg. Cz.; 1 spec. Riu Sadu [Riu Sadu, Cindrel Mts, SB], 15.08.1911, leg. Cz.; 1 spec. Rotterturm Pas [Turnu Roşu Pass, SB], without collection date, leg. Da.

EWC: 1 spec. cabana ? [hut ?], without data; 1 spec. without data; 2 specs Herkulesbad [Băile Herculane, CS], 18.07.1936, leg. Anonymous; 1 spec. Zood-Tal [Sadu, SB], 17.07.1938, leg. Anonymous.

ESC: 2 specs Valea Ştezii, Răşinari [Şteaza Valley, Răşinari, SB], 14.08.1956, leg. E.S.; 2 specs Herkulesbad [Băile Herculane, CS], 6-10.07.1970, leg. E.S.

\section{DISCUSSIONS}

The analyzed species are part of seven faunal types; most of the analyzed taxa belong to the Carpathian faunal type (13 taxa).

The majority of these endemic taxa have a wide range of distribution, such as Erebia epiphron transsylvanica, Pieris bryoniae carpathensis, while others subspecies have a vicarious distribution. E. pandrose roberti is distributed in Northern, North-East Carpathians and Tatra Mountains (Cupedo, 2007) and 
E. pandrose cibinica in the Southern Carpathians, Erebia manto manto is more widespread, and, on the other hand, the endemic Erebia manto trajanus occurs only in the Eastern and Southern Carpathians (Rákosy, 1997; Burnaz, 2008).

There are also endemic subspecies that are restricted to a single or a small number of mountain ranges; Erebia melas carpathicola can be found only in the Haghimaş - Ciucaş Mountains (König, 1982), Erebia melas runcensis is restricted to Apuseni Mountains (Vălişoarei-Aiud and Runcului gorges), Erebia pronoe regalis occurs just in the Bucegi Mountains (König, 1982); in the collection we can find seven specimens from this taxa sampled from Piatra Craiului Mts, B. pales carpathomeridionalis in Bucegi, Piatra Craiului and Făgăraş Mountains and Erebia cassioides neleus can be found in the western part of the Southern Carpathians, especially in the Retezat, Țarcu-Godeanu and Cernei Mountains (König, 1982; Rákosy, 1997; Burnaz, 2008).

In addition the Alpine and Arctic-Alpine faunal types are represented in the Entomological collections with four endemic subspecies. Three of them are representatives of the genus Glacies: the endemic subspecies Glacies coracinus dioszeghyi was described by Schmidt on the basis of the specimens collected by Diószeghy in the Retezat Mountains (Diószeghy, 1933-1934) (the only site in which this subspecies can be found), Glacies noricana carpathica is located in Retezat and Bucegi mountains and Glacies canaliculata schwingenschussi, in the higher mountains of the whole Carpathian chain (Varga, 2010).

South-Continental species are also present in the collections, such as Pseudophilotes bavius hungaricus Diószeghy, 1913. They are spread just in the Transylvanian Plateau, inside the Romanian Carpathians (König, 1992). In the Lepidoptera Collections, we encounter specimens only from the Steppe Reserve from Sarba Valley (Zakel Hill, Slimnic, Sibiu County). Other South-continental subspecies have the distribution range outside the Romanian Carpathians, Tomares nogelii dobrogensis, the only Lepidoptera subspecies of this kind, present in the collections of the museum, was spread just in some small areas from Dobrogea (Rákosy, 1997). Unfortunately this subspecies has disappeared from Romania (van Sway et al., 2010; Marcu \& Rákosy, 2002).

Not all of the subspecies contained in this paper have a well-defined taxonomic status, some endemic subspecies remaining uncertain. E. pandrose cibinica and E. pandrose roberti are closely related, these two subspecies share a pronounced design, especially of the marginal elements, on the hind wing underside (Cupedo, 2007), and Euphydryas maturna partiensis is considered consubspecific with nominotypical maturna (Abadjiev \& Beshkov, 2003). Erebia cassioides neleus is a problematic subspecies as well. The Balkan populations of Erebia cassioides are considered a different species by Albre et al. (2008), Erebia macedonica, and Transylvanian populations are closely related with this population as well as the Alpine populations, raising the question if the ssp neleus is not a different species.

The Macrolepidoptera specimens were collected from nothing less than 118 sampling sites. The number of endemic taxa, according to the data stored in the museum's collection, is higher in some of the collecting sites than in other sites. These sites, located especially in the Eastern and Southern Carpathian Mountains, highlight these zones as core areas for survival and autochthonous evolution for invertebrate groups of limited mobility as considered by Varga $(2003,2010)$ and Burnaz (2008).

In the Lepidoptera Collections the highest abundance of endemic taxa, represented by the numerous number of the species sampled from areas which are 
considered important endemic zones, is encountered in the Southern Carpathians, from Făgăraş Mts with 11 endemic taxa sampled, followed by Retezat Mts, with nine sampled taxa, followed by Piatra Craiului and Cindrel Mts both with six taxa, Parâng and Bucegi Mts each with five sampled taxa.

The number of specimens varies in different sample sites as follows: Făgăraş Mts (91 specs) followed by Retezat (55 specs), Piatra Craiului Mts (36 specs), Cindrel Mts (23 specs), Bucegi Mts, Parâng Mts, Borsec and Bistriţa Bârgăului region (Harghita County).

The high number of endemic taxa and of the specimens sampled from these areas not only confirms the high diversity of endemic taxa, but also outlines once more the core areas for the Lepidoptera and other endemic insect species (Varga, 2010). This is another reason why these areas should be protected.

Some of the endemic subspecies are protected by the Romanian laws (e.g. Habitats Directive). Boloria pales carpathomeridionalis, Erebia gorge fredericikoenigi, Erebia melas carpathicola, Erebia melas runcensis, Erebia sudetica radnaensis, and Pseudophilotes bavius hungaricus are included in the annexes of the directive (Rákosy, 2005).

Along with the other Entomological Collections from the Natural History Museum, the Lepidoptera Collections have an important value (from a historic, scientific and biogeographic point of view). The numerous species, endemic and rare taxa but also wide spread, from Romania and other countries, preserved in these collections, enhance the existing data concerning their geographical distribution, and they may be subjects for creating databases for future studies in conservation, distribution, fauna and biodiversity.

\section{ACKNOWLEDGEMENTS}

The authors wish to thank Prof. Dr. László Rákosy and Prof. Dr. Zoltán Varga for the support in text review and for the valuable comments, also to Popa Rareş, Tăușan Ioan and AnaMaria Mesaroş for linguistique correction of the manuscript. We also thank the anonymous referees for their constructive comments on the manuscript.

This work was possible with the financial support of the Sectoral Operational Programme for Human Resources Development 2007-2013, co-financed by the European Social Fund, under the project number POSDRU/107/1.5/S/76841 with the title „Modern Doctoral Studies: Internationalization and Interdisciplinarity".

\section{SUBSPECII DE MACROLEPIDOPTERE ENDEMICE ÎN COLECŢIILE MUZEULUI DE ISTORIE NATURALĂ DIN SIBIU (ROMÂNIA)}

\section{REZUMAT}

Lucrarea prezintă date referitoare la subspeciile de Macrolepidoptere endemice din colecţiile entomologice ale Muzeul de Istorie Naturală din Sibiu. În aceste colecţii sunt prezente 382 specimene de Macrolepidoptere endemice încadrate în 22 subspecii. Printre subspeciile cuprinse în această lucrare se numără şi Erebia melas runcensis König, 1965, Erebia melas carpathicola Popescu-Gorj \& Alexinschi, 1959, Erebia pronoe regalis Hormuzachi, 1937 şi Erebia cassioides neleus (Freyer, 1833), specii a căror distribuție se rezumă la doar câteva masive muntoase. Majoritatea specimenelor au fost colectate din habitatele din Munţii Carpaţi, în special din Carpaţii Meridionali şi Carpaţii Occidentali. Astfel această lucrare aduce contribuţii la distribuţia subspeciilor endemice cuprinse în ea dar şi evidenţiază zonele endemogeneratoare şi endemoconservatoare din care provin. 


\section{LITERATURE CITED}

ABADJIEV, S., S. BESHKOV, 2003 - More about Euphydrias maturna in Bulgaria (Lepidoptera: Nymphalidae, Nymphalinae). Phegea, 31 (1): 33-37.

ALBRE, J., C. GERS, L. LEGAL, 2008 - Molecular phylogeny of the Erebia tyndarus (Lepidoptera, Rhopalocera, Nymphalidae, Satyrinae) species group combining CoxII and ND5 mitochondrial genes: A case study of a recent radiation. Molecular Phylogenetics and Evolution, 47 (1): 196-210.

BARTHA, V., 1933 - Die Psodos-Arten des Retyzat-Gebirges. Mitteilungen der Münchner Entomologischen Gesellschaft, 23: 37-42.

BURNAZ, S., 2008 - Endemite şi specii rare în colecţia de lepidoptere a Muzeului Civilizaţiei Dacice şi Romane Deva (judeţul Hunedoara, România). Oltenia, Studii şi comunicări, Ştiinţele Naturii, 24: 130-138. (in Romanian)

CUPEDO, F., 2007 - Geographical variation and pleistocene history of the Erebia pandrose - sthennyo complex. Nota Lepidopterologica, 30: 329-353.

CUVELIER, S., V. DINCĂ, 2007 - New data regarding the butterflies (Lep.: Rhopalocera) of Romania, with additional comments (general distribution in Romania, habitat preferences, threats and protection) for ten localized Romanian species. Phegea, 35 (3): 93-115.

CZEKELIUS, D., 1917 - Beiträge zur Schmmetterlingsfauna Siebenbürgens. Verhandlungen und Mitteilungen des Siebenbürgischen Vereins fur Naturwissenschaften zu Hermannstadt, 67: $1-57$.

CZEKELIUS, D., 1922 - Beitrage zur Schmetterlingsfauna Siebenbürgens. Verhandlungen und Mitteilungen des Siebenbürgischen Vereins fur Naturwissenschaften zu Hermannstadt, 71: 71-77.

DE JONG, Y. S. D. M., 2010 - Fauna Europaea version 2.4. Web Service available online at http:// www.faunaeur.org.

DIÓSZEGHY, L., 1933-1934 - Die Lepidopterenfauna des Retezatgebirges. Nachtrag I. Verhandlungen und Mitteilungen des Siebenbürgischen Vereins fur Naturwissenschaften zu Hermannstadt, 83-84: 107-126.

GASTON, K. J., J. I. SPICER, 1998 - Biodiversity: an introduction. Blackwell Science, Oxford. 113 pp.

KENYERES, Z., I. A. RÁCS, Z. VARGA, 2009 - Endemism hot spots, core areas and disjunctions in European Orthoptera. Acta Zoologica Cracoviensia, 52B (1-2): 189-211.

KÖNIG, F., 1982 - Specii de lepidoptere montane, sub-alpine, alpine și boreo-alpine din Carpații Românești. Societatea de Ştiinţe biologice din R.S.R. filiala Reghin, Studii şi Comunicări, 2: 229-236. (in Romanian)

KÖNIG, F., 1992 - Morphologische, biologische und ökologische Daten über Philotes bavius hungarica Diószeghy, 1913 (Lepidoptera: Lycaenidae). Entomologische Zeitschrift mit Insektenboerse, Essen, 102 (9): 168-172.

LERAUT, P., 2006 - Moths of Europe. Saturnids, Lasiocampids, Hawkmoths, Tiger Moths. N.A.P. Editions, Verrières le Buisson, 1: 1-395.

LERAUT, P., 2009 - Moths of Europe. Geometrid Moths. N.A.P. Editions, Verrières le Buisson, 2: $1-808 \mathrm{pp}$.

MARCU, A., L. RÁKOSY, 2002 - Catalogul colecţiei de lepidoptere "Dr. Vladimir Olaru" din Complexul Muzeal de Ştiinţele Naturii Galaţi. Societatea Lepidopterologică Română, ClujNapoca, 172 pp. (in Romanian)

NICULESCU, E. V., 1961 - Insecta. Lepidoptera. Family Papilionidae. In: Fauna Republicii Socialiste Române, XI (5). Edit. Academiei Republicii Socialiste Române, Bucureşti, 11 (5): 1-120. (in Romanian)

NICULESCU, E. V., 1963 - Insecta. Lepidoptera. Family Pieridae (Fluturi). In: Fauna Republicii Socialiste Române. Edit. Academiei Republicii Socialiste Române, Bucureşti, 11 (6): 1-202. (in Romanian)

NICULESCU, E. V., 1965 - Insecta. Lepidoptera. Family Nymphalidae. In: Fauna Republicii Socialiste Române. Edit. Academiei Republicii Socialiste Române, Bucureşti, 11 (7): 1-361. (in Romanian)

PASCU, M., E. SCHNEIDER, 1998 - Colecţiile entomologice ale Muzeului de Istorie Naturală din Sibiu. Studii şi Comunicări. Muzeul Brukenthal, 27: 201-218. (in Romanian)

RÁKOSY, L., 1996 - Die Noctuiden Rumäniens (Lepidoptera: Noctuidae). Staphia, Linz, 46: 1-648.

RÁKOSY, L., 1997 - Die endemischen Lepidopteren Rumäniens. Entomologica Romanica, ClujNapoca, 2: 59-81.

RÁKOSY, L., 2003 - Lista roşie pentru fluturii diurni din România. Buletin Informativ, Societatea lepidopterologică română, 13 (1-4): 9-26. (in Romanian) 
RÁKOSY, L., 2005 - U.E. şi legislaţia pentru protecţia lepidopterelor din România. Buletinul de Informare Entomologică, Cluj-Napoca, 16 (3-4): 89-96. (in Romanian)

RÁKOSY, L., M. GOIA, Z. KOVÀCS, 2003 - Catalogul Lepidopterelor României / Verzeichnis der Schmetterlinge Rumäniens. Societatea Lepidopterologică Română, Cluj-Napoca, 446 pp. (in Romanian)

RÁKOSY, L., K. PECSENYE, C. MIHALI, A. TÓTH, Z. VARGA, 2012 - Taxonomic review of Euphydryas maturna (Linnaeus, 1758) (Lepidoptera, Nymphalidae) with description of a new subspecies from Dobrogea (Romania) and notes on conservation biology. Acta Zoologica Academiae Scientiarum Hungaricae, 58 (2): 145-161.

SCHNEIDER, E., 1984 - Die Gross-Schmetterlinge der Sammlung Dr. V. Weidell. Ein Beitrag zur Faunistik der Lepidopteren Südsiebenbürgens und angrenzender Gebiete. Studii şi comunicări, Științele Naturii, Muzeul Brukenthal, Sibiu, 26: 289-316.

SCHNEIDER, E., $2003^{\circ}$ - Die Schmetterlingssammlung Heinrich von Hannenheims im Naturwissenschaftlichen Museum in Hermannstadt (Sibiu). Studii și comunicări, Științele Naturii, Muzeul Brukenthal, Sibiu, 28: 201-230.

TOLMAN, T., R. LEWINGTON, 2009 - Collins Butterfly Guide. The most complete guide to the Butterflies of Britain and Europe. Harpes Collins Publishers, London, $384 \mathrm{pp}$.

VAN SWAAY, C., A. CUTTELOD, S. COLLINS, D. MAES, M. LOPEZ MUNGUIRA, M. ŠAŠIĆ, J. SETTLE, R. VEROVNIK, T. VERSTRAEL, M. WARREN, M. WIEMERS, I. WYNHOF, 2010 - European Red List of Butterfies. Council of Europe Publishing, Luxembourg, 60 pp.

VARGA, Z., 1999 - Die Erebien der Balkanhalbinsel und Karpaten IV. Übersicht der subspezifischen Gliederung und der Verbreitung der Erebia-Arten in der Balkanhalbinsel und in den Karpaten (Lepidoptera, Nymphalidae, Satyrinae). Entomologica romanica, 3 (1998): 15-29.

VARGA, Z., 2002 - Die Erebien der Balkanhalbinsel und Karpaten IV. Übersicht der subspezifischen Gliederung und der Verbreitung der Erebia Dalman, 1816 - Arten (Lepidoptera: Nymphalidae: Satyrinae) in der Balkanhalbinsel und in den Karpaten (II. Teil). Entomologica Romanica, 6 (2001): 5-39.

VARGA, Z., 2003 - Post-glacial dispersal strategies of Orthoptera and Lepidoptera in Europe and in the Carpathian basin. Proceedings of the 13 Colloquium of EIS, Leiden, 2001: 93-105.

VARGA, Z., 2010 - Extra-Mediterranean refugia, post-glacial vegetation history and area dynamics in Eastern Central Europe. Pp. 57-90. In: J. Habel, Th. Assmann (eds), Relict species: Phylogeography and Conservation Biology. Springer-Verlag Berlin Heidelberg, 449 pp.

Received: August 6, 2012

Accepted: June 12, 2013
Török Sergiu-Cornel

Babeş-Bolyai University Faculty of Biology and Geology

Clinicilor str. 5-7, 400006 Cluj-Napoca, Romania.

e-mail: ser.torok@yahoo.com

Cuzepan Gabriela

Brukenthal National Museum, Natural History Museum str. Cetăţii no. 1, 550166 Sibiu, Romania.

e-mail: gabrielacuzepan@gmail.com 